\title{
Ausstellung zum Thema Früchte und Ausbreitung im Botanischen Garten Osnabrück
}

\author{
Martin Husemann
}

\begin{abstract}
Fruits play an important role in protection and dispersal of plant seeds. With their specific contents fruits play a major role in human nutrition and medicine. Carpology deals with the morphology, physiology and ecology of fruits. Since summer 2007 the Botanical Garden of Osnabrück illustrates the diversity and beauty of fruits in an exposition of its carpological collection.
\end{abstract}

\section{Zusammenfassung}

Früchte sind für Schutz und Verbreitung der Samen von Pflanzen von großer Bedeutung. Mit ihren spezifischen Inhaltsstoffen haben sie für den Menschen eine besondere Bedeutung in der Ernährung und der Medizin. Die Karpologie befasst sich mit der Morphologie, Physiologie und Ökologie von Früchten und Fruchtständen. Um diese Vielfalt und Schönheit zu illustrieren, stellt der Botanische Garten Osnabrück seit dem Sommer 2007 seine Sammlung heimischer und exotischer Früchte aus.

\section{Einleitung}

Schon die ersten Menschen beschäftigten sich mit den Früchten der Pflanzen. Sie nutzten die vielfältigen Geschenke der Natur als Nahrungsressource, Heilmittel und Schmuck. Bis heute haben Früchte und Samen ihre Bedeutung für den Menschen nicht verloren.

In der Fruchtkunde (Karpologie) wird die Frucht als Blüte im Zustand der Samenreife definiert. Ihre Aufgabe ist es, die Samen der Pflanze zu schützen und bei der Ausbreitung zu helfen. Im Zeitalter der molekularen Forschung erscheint es vielleicht altmodisch, Obst und Gemüse in einer Ausstellung zu präsentieren. In Anbetracht der wichtigen Rolle der Früchte ist es durchaus sinnvoll, Besuchern eines Botanischen Gartens die große Vielfalt, Bedeutung und Funktionsweise ausgewählter Früchte vorzustellen.

2. Früchte in der Ernährung und der Medizin Als Nahrungsmittel spielen Früchte eine wichtige Rolle. Getreide gehören zu unseren wichtigsten Nahrungsmitteln. Allerdings unterscheiden sich die unterschiedlichen Getreidearten regional in ihrer Bedeutung. In Teilen von Asien, Afrika und Lateinamerika ist Reis (Oryza sativa) die Ernährungsgrundlage. In vielen Regionen wird er durch verschiedene Hirsen (Sorghum, Panicum, Setaria) ergänzt. In Lateinamerika ist Mais (Zea mays) besonders wichtig, während in Eu- ropa und Nordamerika der Weizen (Triticum aestivum) das wichtigste Getreide ist. Während Getreide vor allem Kohlenhydrate liefert, stellen Hülsenfrüchte wie die Sojabohne (Glycine max) oder die Erbse (Pisum sativum) wichtige Proteinquellen dar (vgl. dazu auch Franke et al. 2007).

Unter Obst versteht man eine saftig-fleischige Frucht, die roh verzehrt werden kann. Obst liefert neben verschiedenen Zuckern vor allem wichtige Vitamine und Spurenelemente. Unter Gemüse verstehen wir außer den Früchten von Pflanzen (z.B. Gurke, Cucumis sativus) z. B. auch deren Sprossachsen (z. B. Bambus), Sprossknollen (z. B. Kartoffel, Solanum tuberosum), Rüben (z. B. Möhre, Daucus carota) oder auch die ganze Pflanze (z. B. Porree, Allium porrum).

Die Inhaltsstoffe von Pflanzen spielen auch in der Medizin eine bedeutende Rolle. Da die Naturheilkunde in den letzten Jahren einen großen Aufschwung erlebt, erlangen die verschiedenen Arzneipflanzen wieder neue Bedeutung. Oft sind Wirkstoffe in Früchten konzentriert. Aus unreifen Früchten der Papaya (Carica papaya) beispielsweise wird ein Milchsaft gewonnen. Dieser enthält Protein-spaltende Enzyme, vor allem Papain und Chymopapain. Papaya-Präparate dienen als Verdauungshilfe, als Wurmmittel und in Verbindung mit ähnlichen Enzymen tierischer Herkunft zur Langzeitbehandlung bei Tumoren. 

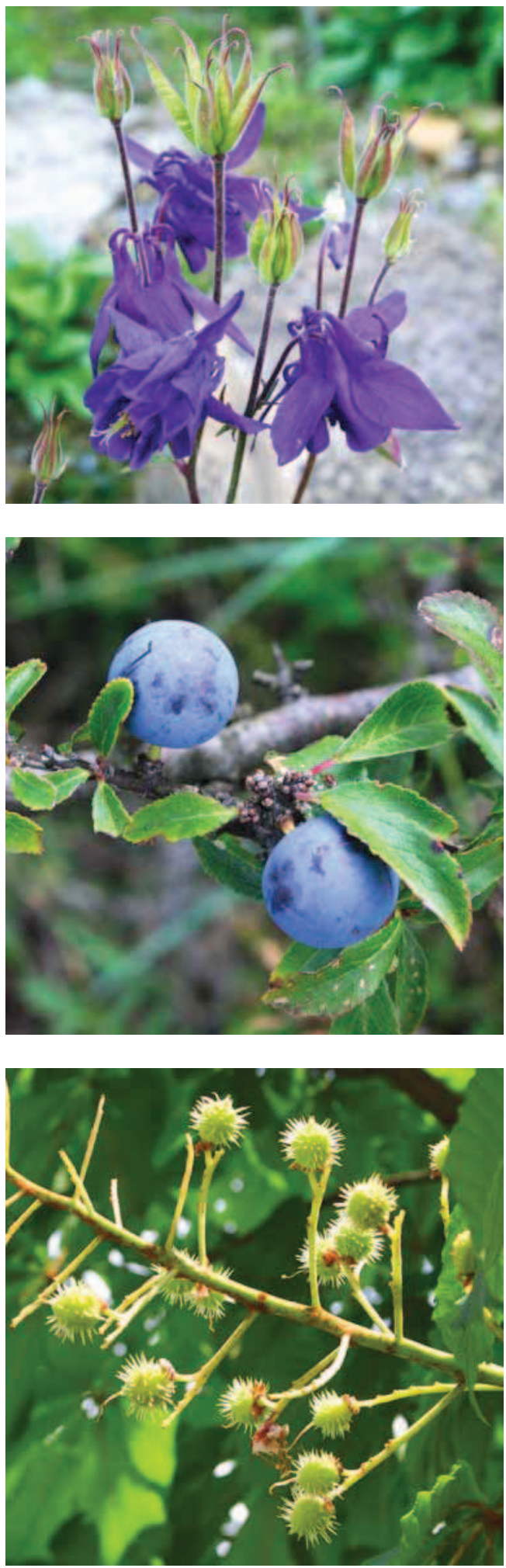

\section{Früchte in der Botanik}

Der Karpologe (Fruchtkundler) beschreibt Morphologie und Anatomie der Früchte, ordnet sie Typen zu, die sich in der Entstehung und in der Beteiligung von verschiedenen Organen der Pflanze am Fruchtaufbau unterscheiden, und untersucht die Ausbreitung der Samen, soweit die Frucht daran beteiligt ist.

\subsection{Fruchttypen}

Man unterscheidet beispielsweise Hülse, Schote, Balgfrucht (z. B. Akelei, Aquilegia), Nussfrucht, Steinfrucht (z. B. Schlehe, Prunus spinosa), Karyopse, Achäne, Beere und Kapsel (z. B. Rosskastanie, Aesculus hippocastanum). Zudem kommen verschiedene Sammelfrüchte vor. Zwar gibt es viele Überlappungen in der Umgangssprache, doch meint der Botaniker häufig etwas anderes als der Laie. So sind Gurke und Kürbis im botanischen Sinne Beeren, die Erdbeere ist eine Sammelnüsschenfrucht und die Kokosnuss eine Steinfrucht.

\subsection{Ausbreitung}

Um die Art dauerhaft zu erhalten, kann es notwendig sein, neue Lebensräume zu erschließen. Da die Pflanze selbst aber unbeweglich ist, müssen spezielle Organe diese Aufgabe übernehmen. Oft ist die Frucht entscheidend an der Ausbreitung des Samens beteiligt. Im Laufe der Evolution haben sich im Pflanzenreich verschiedene Ausbreitungsmechanismen entwickelt (vgl. auch Maidhof 2006, 2007, LeINs \& ERbar 2008).

Die Diasporen (Früchte oder Samen) vieler Pflanzen werden durch Tiere ausgebreitet (Zoochorie). Bei der Endozoochorie werden Früchte und Samen gefressen und im keimfähigen Zustand wieder ausgeschieden (z. B. Walderdbeere, Fragaria vesca). Exozoochor verbreitete Früchte haben Kleb- oder Klettvorrich-

Abb. 1 (oben): Schüttelbalgfrüchte einer Akelei.

Abb. 2 (Mitte): Die Steinfrüchte der Schlehe werden in der Naturheilkunde genutzt, aber auch zu Marmelade und Likör verarbeitet.

Abb. 3 (unten): Unreife Kapselfrüchte der Rosskastanie. 
tungen, mit denen sie sich im Fell von Tieren verankern, bis sie an anderer Stelle wieder abfallen. Beispiel sind die Klettfrüchte des Kletten-Labkrauts (Galium aparine). Myrmekochorie ist die Ausbreitung durch Ameisen. Manche Früchte oder Samen tragen ein fettreiches Körperchen, das Elaiosom, das die Ameisen dazu bewegt, die Diasporen zu ihrem Nest zu tragen.

Auch die abiotischen Faktoren Wind und Wasser werden von den Pflanzen zur Ausbreitung genutzt. Unter Hydrochorie versteht man die Ausbreitung durch Wasser wie z. B. bei der Kokosnuss (Cocos nucifera). Die Früchte fallen nach der Reife ins Wasser und werden weggetragen, um an anderer Stelle zu keimen.

Anemochore Pflanzen werden durch Wind ausgebreitet. Anemochore Diasporen haben oft haarige oder häutige Anhängsel, die ihre Oberfläche flügelartig vergrößern und damit das Flugverhalten verbessern. Bei anderen Pflanzen sind die Samen sehr klein und fliegen auch ohne spezielle Flugorgane gut (z. B. viele Orchideen). Eine bekannte, vom Wind ausgebreitete Art ist der Löwenzahn (Taraxacum officinale). Bei verschiedenen Ahorn-Arten (Acer) bilden die Früchte flügelartige Auswüchse. Daneben gibt es blasig aufgetriebene Früchte, die wie ein Ballon fliegen (z. B. Blasenstrauch, Colutea arborescens). Beim Klatschmohn (Papaver rhoeas) werden die samentragenden Kapseln vom Wind geschüttelt, wodurch die Samen ausgestreut werden. Bei Steppenrollern wird nahezu die gesamte Pflanze ausgebreitet. Ein typisches Beispiel ist die Gemeine Sichelmöhre (Falcaria vulgaris), bei der die Pflanze kurz über der Wurzel abbricht. Die oberirdischen Pflanzenteile rollen über ebene Flächen hinweg, wobei die Früchte gleichmäßig verteilt werden.

Abb. 4 (oben): Die Sammelnüsschenfrüchte der Walderdbeere werden von Tieren gefressen, die einzelnen Nüsschen werden wieder ausgeschieden.

Abb. 5 (Mitte): Ausschnitt aus der Oberfläche einer Haftfrucht des Japanischen Klettenkerbels (Torilis japonica), rasterelektronenmikroskopische Aufnahme.

Abb. 6 (unten): Der Wiesenbocksbart (Tragopogon pratensis) hat anemochore Früchte (Achänen), die wie Fallschirme fliegen.
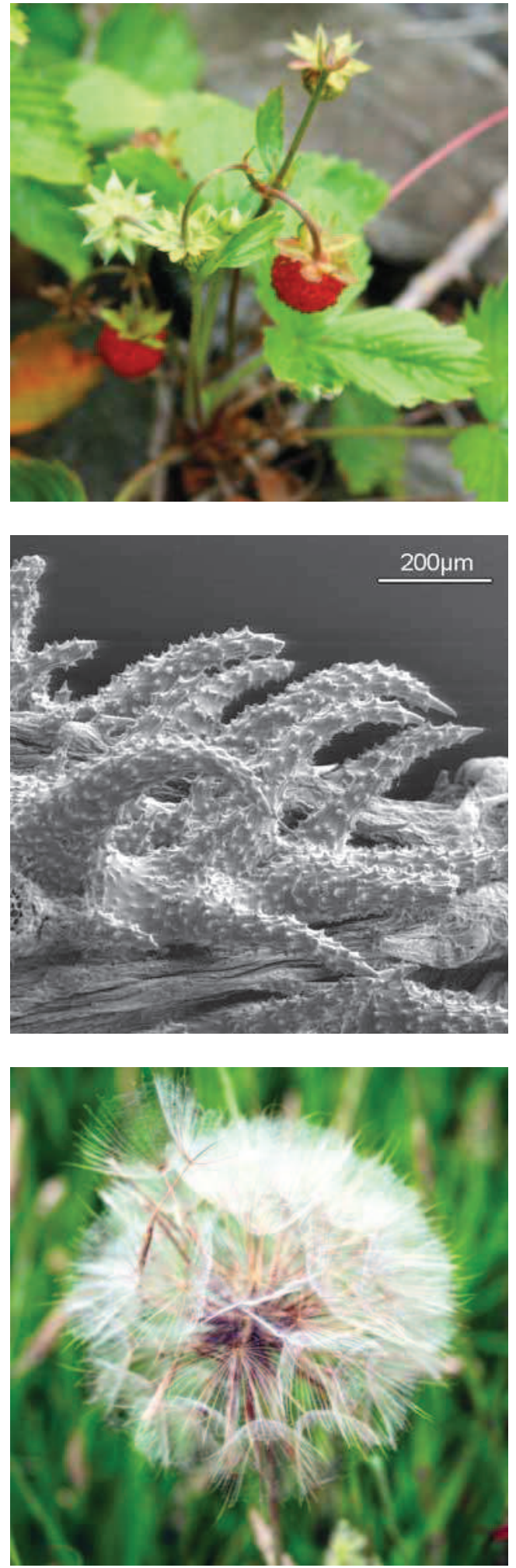

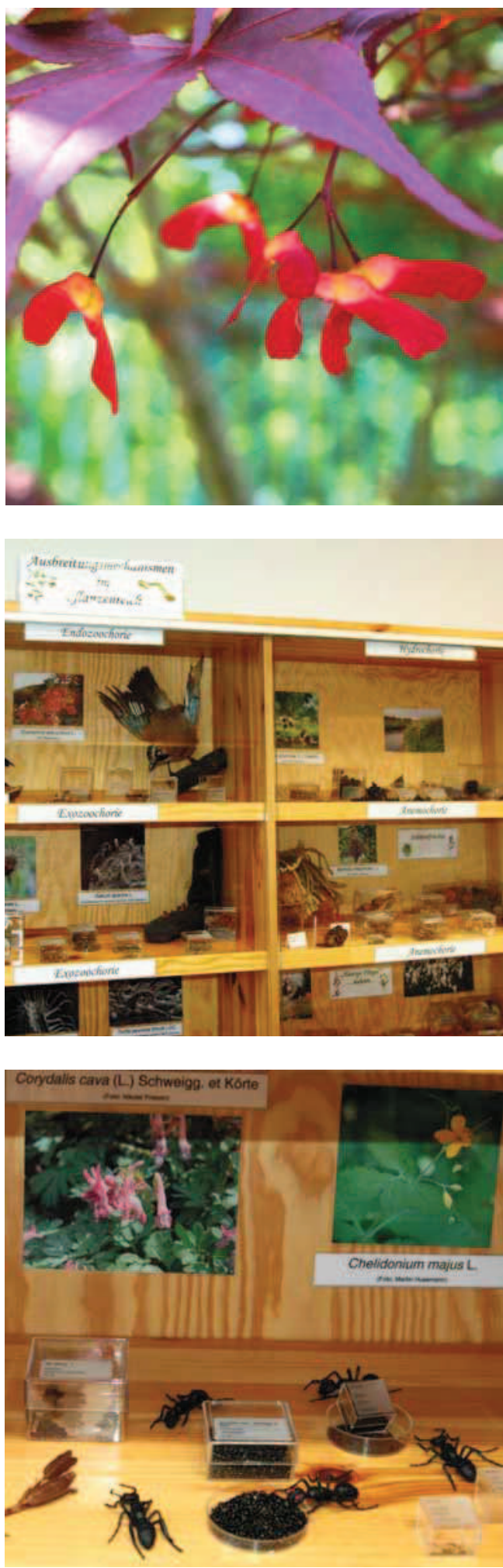

Ballochorie ist die Ausbreitung von Diasporen durch Schleudermechanismen. Während bei so genannten Saftschleuderern die Energie zum Ausschleudern der Samen aus dem Turgordruck lebender Gewebe stammt, trocknen bei den Austrocknungsschleuderern unterschiedlich strukturierte Gewebeschichten aus, zwischen denen es beim Trocknen zu Spannungen kommt. Diese entladen sich, wenn Sollbruchstellen reißen, und durch frei werdende Energie werden die Samen ausgeschleudert wie z. B. beim Springkraut (Impatiens).

\section{Ausstellung der karpologischen Samm- lung im Botanischen Garten Osnabrück}

Um den Besuchern zusätzlich zum vielfältigen Angebot des Botanischen Gartens eine weitere Möglichkeit anzubieten, die große Vielfalt des Pflanzenreichs, insbesondere der Früchte, kennen zu lernen, stellt der Botanische Garten Osnabrück seit dem Sommer 2007 einen Teil seiner karpologischen Sammlung aus. Diese umfasst zurzeit die Früchte von über 900 verschiedenen heimischen und exotischen Pflanzen. Die Exponate sind nach den oben beschriebenen Kriterien sortiert. Während also ein Teil der Ausstellung den botanischen Fruchttypen gewidmet ist, sind in einem anderen Bereich einige der im Pflanzenreich realisierten Ausbreitungsmechanismen anhand von Beispielen vorgestellt. Ein dritter Teil der Ausstellung behandelt Früchte im Zusammenhang mit Ernährung und Medizin. Zusätzlich sind einige besonders schöne oder beeindruckende Stücke wie die Riesenhülse (Entada phaseoloides) oder die Früchte des Heuschreckenbaumes (Hymenaea coubaril) ausgestellt. Auch Früchte invasiver Pflanzen werden gezeigt.

Zusätzlich zu der Ausstellung ist ein Beiheft in Arbeit, das weitere Informationen zu den übergeordneten Themen und Detailangaben zu

Abb. 7 (oben): Die Früchte des Fächerahorns werden vom Wind mehrere Meter von der Mutterpflanze weggeweht.

Abb. 8 (Mitte): Eine Vitrine der Ausstellung ist den Ausbreitungsstrategien von Pflanzen gewidmet.

Abb. 9 (unten): Vitrine zum Thema Myrmekochorie. 


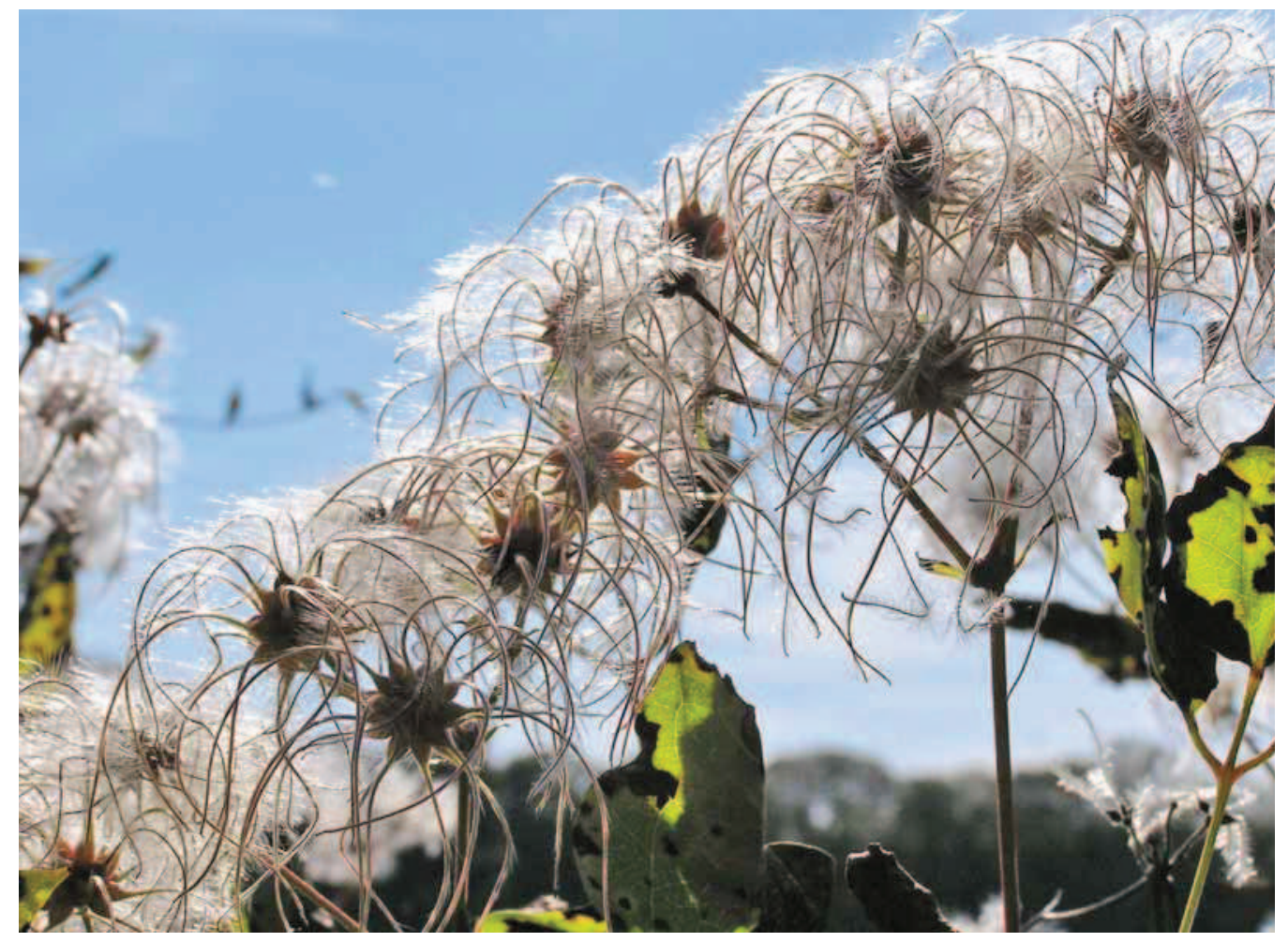

einzelnen ausgewählten Pflanzen bieten wird. Das Heft kann nach Erscheinen für einen geringen Unkostenbeitrag im Sekretariat des Botanischen Gartens Osnabrück erworben werden.

\section{Dank}

Besonderer Dank gilt allen, die an der Ausstellung mitgearbeitet haben, allen Sammlern und insbesondere Heiner Flinks, Katharina Berger, Miriam Hanitzsch, Ulrich Rösemann, PD Dr. Nikolai Friesen und Dr. Peter BORGMANN für die kritische Begutachtung des Manuskripts.
Literatur

Franke, W., Lieberei, R. \& Reisdorff, C. 2007: Nutzpflanzenkunde. 11. Aufl. - Stuttgart.

Leins, P. \& Erbar, C. 2008: Blüte und Frucht. Morphologie, Entwicklungsgeschichte, Phylogenie, Funktion, Ökologie, 2. Aufl. - Stuttgart.

Maidhof, A. 2006: Morphologische Beobachtungen über Verbreitungseinrichtungen an Samen und Früchten von bedecktsamigen Pflanzen, Teil 1: Einrichtungen zur Windverbreitung. - Mikrokosmos 95: 369-375. Maidhof, A. 2007: Morphologische Beobachtungen über Verbreitungseinrichtungen an Samen und Früchten von bedecktsamigen Pflanzen, Teil 2: Einrichtungen zur Tier-, Wasser- und Selbstverbreitung. - Mikrokosmos 96: $18-24$. 